Pei-Xuan Li*, Wei Hui and Qin-Qin Jing

\title{
Crystal structure of methyl 4-\{[4- \\ (4-cyanobenzamido)phenyl]amino\}benzofuro \\ [2,3-d]pyrimidine-6-carboxylate, $\mathrm{C}_{26} \mathrm{H}_{17} \mathrm{~N}_{5} \mathrm{O}_{4}$
}

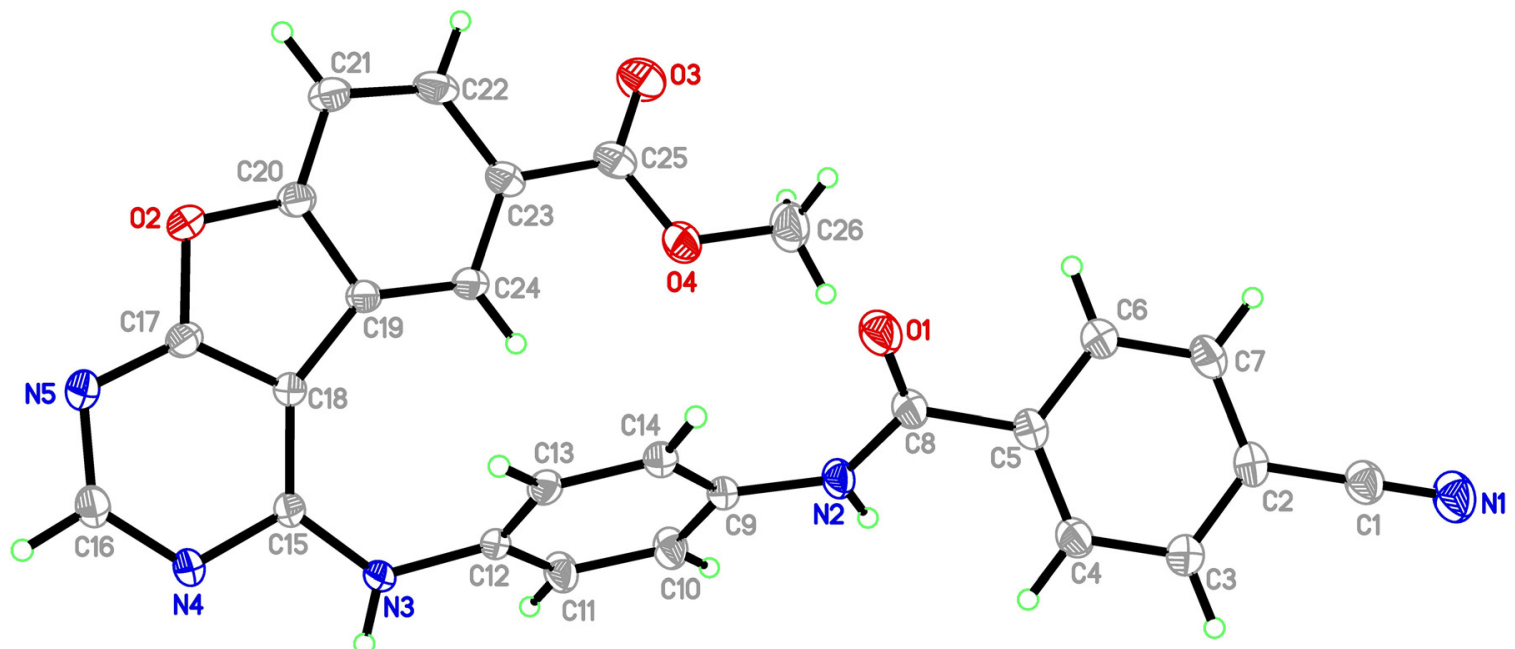

https://doi.org/10.1515/ncrs-2022-0013

Received January 13, 2022; accepted February 7, 2022;

published online February 24, 2022

\section{Abstract}

$\mathrm{C}_{46} \mathrm{H}_{38} \mathrm{~N}_{10} \mathrm{~S}_{2}$, triclinic, $P \overline{1}$ (no. 2), $a=8.9297(12) \AA$, $b=10.9926(15) \AA, c=12.6758(18) \AA, \alpha=104.532(2)^{\circ}$, $\beta=96.312(2)^{\circ}, y=112.398(2)^{\circ} V=1083.8(3) \AA^{3}, Z=2$, $R_{g t}(F)=0.0398, w R_{\text {ref }}\left(F^{2}\right)=0.1195, \mathrm{~T}=296(2) \mathrm{K}$.

CCDC no.: 1906988

The molecular structure is shown in the figure. Table 1 contains crystallographic data and Table 2 contains the list of the atoms including atomic coordinates and displacement parameters.

*Corresponding author: Pei-Xuan Li, College of Safety Science and Engineering, Xi'an University of Science and Technology, Xi'an 710054, China, E-mail:39871439@qq.com. https://orcid.org/00000003-0698-8761

Wei Hui, China Northwest Architecture Design and Research Institute Co. LTD, Xi'an 710018, China

Qin-Qin Jing, Yanan Energy and Chemical Group Co., LTD, Yan'An, China

Ә Open Access. ( 2022 Pei-Xuan Li et al., published by De Gruyter. ((c)) BY License.
Table 1: Data collection and handling.

\begin{tabular}{ll}
\hline Crystal: & Colorless block \\
Size: & $0.26 \times 0.23 \times 0.20 \mathrm{~mm}$ \\
Wavelength: & Mo $K \alpha$ radiation $(0.71073 \AA$ A $)$ \\
$\mu:$ & $0.10 \mathrm{~mm}^{-1}$ \\
Diffractometer, scan mode: & Bruker APEX-II, $\varphi$ and $\omega$ \\
$\theta_{\max }$, completeness: & $25.0^{\circ},>99 \%$ \\
$N\left(h k l_{\text {measured }}, N\left(h k l_{\text {unique }}, R_{\text {int }}:\right.\right.$ & $7604,3800,0.021$ \\
Criterion for $I_{\text {obs }}, N(h k l)_{\text {gt }}:$ & $I_{\text {obs }}>2 \sigma\left(I_{\text {obs }}\right), 2865$ \\
$N(\text { param })_{\text {refined: }}:$ & 317 \\
Programs: & CrysAlis \\
\hline
\end{tabular}

\section{Source of material}

The methyl 4-[(4-aminophenyl)amino]benzofuro[2,3- $d]$ pyrimidine-6-carboxylate 7 ( $2.0 \mathrm{~g}, 6.0 \mathrm{mmol})$, 4-cyanobenzoic acid (1.15 g, $7.8 \mathrm{mmol}$ ), HATU (systematic name : 1-[Bis(dimethylamino)methylene]-1H-1,2,3-triazolo[4,5-b]pyridinium 3-oxide hexafluorophosphate; $2.96 \mathrm{~g}, 7.8 \mathrm{mmol}$ ) and $\mathrm{Et}_{3} \mathrm{~N}$ $(1.2 \mathrm{~g}, 12.0 \mathrm{mmol})$ were stirred together in dimethylformamide $(15 \mathrm{~mL})$ at room temperature for $7 \mathrm{~h}$. Upon completion of the reaction, the mixture was diluted with saturated sodium carbonate solution $(50 \mathrm{~mL})$ and subsequently extracted with dichloromethane. The extracts were combined, washed with brine, dried over anhydrous $\mathrm{Na}_{2} \mathrm{SO}_{4}$, and concentrated in vacuo. Purification by silica-gel column 
Table 2: Fractional atomic coordinates and isotropic or equivalent isotropic displacement parameters $\left(\AA^{2}\right)$.

\begin{tabular}{|c|c|c|c|c|}
\hline Atom & $x$ & $y$ & $z$ & $U_{\text {iso }}{ }^{*} / U_{\text {eq }}$ \\
\hline 1 & $0.2075(3)$ & $0.9636(2)$ & $0.48804(17)$ & $0.0437(5)$ \\
\hline 2 & $47(3)$ & $.85162(19)$ & $0.52004(16)$ & $.0384(5)$ \\
\hline $\mathrm{C} 3$ & $0.1096(3)$ & $0.7784(2)$ & $0.57219(16)$ & 0.0409 (5) \\
\hline $\mathrm{H} 3$ & 0.0218 & 0.8009 & 0.5863 & $0.049^{*}$ \\
\hline 4 & $0.1272(2)$ & $0.6717(2)$ & $0.60290(16)$ & $0.0399(5)$ \\
\hline $\mathrm{H} 4$ & 0.0517 & 0.6233 & 0.6391 & $0.048^{*}$ \\
\hline $\mathrm{C} 5$ & $0.2565(2)$ & 0.63591 (19) & $0.58034(15)$ & $0.0338(4)$ \\
\hline C6 & $0.3689(2)$ & $0.7075(2)$ & $0.52555(16)$ & $0.0398(5)$ \\
\hline $\mathrm{H} 6$ & 0.4535 & 0.6820 & 0.5081 & $0.048^{\star}$ \\
\hline $\mathrm{C} 7$ & $0.3545(3)$ & $0.8161(2)$ & $0.49726(16)$ & $0.0421(5)$ \\
\hline H7 & 0.4317 & 0.8659 & 0.4628 & $0.050^{*}$ \\
\hline $\mathrm{C} 8$ & $0.2721(2)$ & $0.5168(2)$ & $0.60996(16)$ & $0.0352(4)$ \\
\hline C9 & $0.2368(2)$ & $0.41242(18)$ & $0.76391(15)$ & $0.0306(4)$ \\
\hline 10 & $0.2532(3)$ & $0.4488(2)$ & $0.87935(16)$ & $0.0390(5)$ \\
\hline $\mathrm{H} 10$ & 0.2806 & 0.5402 & 0.9207 & $0.047^{*}$ \\
\hline C11 & $0.2291(3)$ & 0.35066 (19) & $0.93276(16)$ & $0.0386(5)$ \\
\hline $\mathrm{H} 11$ & 0.2416 & 0.3764 & 1.0100 & $0.046^{*}$ \\
\hline 12 & $0.1865(2)$ & $0.21431(18)$ & $0.87256(15)$ & $0.0299(4)$ \\
\hline 13 & $0.1700(2)$ & $0.17780(18)$ & $0.75761(15)$ & $0.0308(4)$ \\
\hline H13 & 0.1401 & 0.0858 & 0.7165 & $0.037^{*}$ \\
\hline C14 & 1974 (2) & $.27580(18)$ & 327 (15) & 0.0309 (4) \\
\hline $\mathrm{H} 14$ & 0.1894 & 0.2505 & 0.6264 & $0.037^{\star}$ \\
\hline 15 & $0.2079(2)$ & $0.01272(18)$ & 0.913 & $0.0285(4)$ \\
\hline C16 & $0.1607(2)$ & -0.20249 (19) & $0.93002(15)$ & $0.0363(5)$ \\
\hline H16 & 0.0955 & -0.2762 & 0.9522 & $0.044^{*}$ \\
\hline 17 & 3759 (2) & -0.10197 (19) & (14) & $0.0312(4)$ \\
\hline C18 & (2) & 0.01644 & 0.8 & $0.0273(4)$ \\
\hline C19 & 0.49 & $0.10971(18)$ & 88 (14) & 0.0289 (4) \\
\hline 20 & $3(2)$ & 0.03535 (19) & (15) & $0.0338(4)$ \\
\hline $\mathrm{C} 21$ & $0.7340(2)$ & 0.0853 & $0.78159(17)$ & 419 (5) \\
\hline $\mathrm{H} 21$ & 1 & & 59 & $0.050^{*}$ \\
\hline $\mathrm{C} 22$ & $0.7884(2)$ & 0.217 & 0.77403 & $0.0419(5)$ \\
\hline $\mathrm{H} 22$ & 0.8884 & 0.2553 & 32 & $0.050^{*}$ \\
\hline $\mathrm{C} 23$ & $7(2)$ & (2) & 0.7 & $0.0352(5)$ \\
\hline $\mathrm{C} 24$ & $0.5483(2)$ & 0.24295 (19) & $0.83136(14)$ & $0.0310(4)$ \\
\hline 124 & 0.4889 & 0.2960 & 0.8483 & $0.037^{*}$ \\
\hline $\mathrm{C} 25$ & $0.7596(2)$ & $0.4320(2)$ & $0.77734(16)$ & $0.0407(5)$ \\
\hline C26 & $0.6936(3)$ & $0.6120(2)$ & $0.7482(2)$ & $0.0580(6)$ \\
\hline $\mathrm{H} 26 \mathrm{~A}$ & 0.8013 & 0.6784 & 0.7937 & $0.087^{\star}$ \\
\hline $\mathrm{H} 26 \mathrm{~B}$ & 0.6130 & 0.6483 & 0.7622 & $0.087^{\star}$ \\
\hline $\mathrm{H} 26 \mathrm{C}$ & 0.6971 & 0.5940 & 0.6706 & $0.087^{\star}$ \\
\hline N1 & $0.1922(3)$ & $1.0516(2)$ & $0.46162(16)$ & $0.0588(5)$ \\
\hline $\mathrm{N} 2$ & $0.2515(2)$ & 0.51604 (15) & $0.71448(13)$ & $0.0361(4)$ \\
\hline $\mathrm{H} 2$ & 0.2468 & 0.5890 & 0.7560 & $0.043^{*}$ \\
\hline N3 & 15244 (19) & $0.11254(15)$ & 0.92789 (13) & $0.0363(4)$ \\
\hline $\mathrm{H} 3 \mathrm{~A}$ & 0.0904 & 0.1148 & 0.9750 & $0.044^{*}$ \\
\hline N4 & 11724 (18) & $-0.09785(15)$ & $0.94456(13)$ & $0.0326(4)$ \\
\hline N5 & $0.2863(2)$ & $-0.21529(16)$ & $0.88753(13)$ & $0.0361(4)$ \\
\hline 01 & 0078 (19) & $0.42996(15)$ & $0.54474(12)$ & $0.0510(4)$ \\
\hline 02 & $0.51634(16)$ & -0.09451 (13) & $0.82680(11)$ & $0.0377(3)$ \\
\hline 03 & 0.89676 (19) & $0.49024(17)$ & $0.76328(16)$ & $0.0688(5)$ \\
\hline 04 & $0.64762(17)$ & $0.48432(14)$ & $0.77534(12)$ & $0.0472(4)$ \\
\hline
\end{tabular}

chromatography provided $2.13 \mathrm{~g}$ the desired product. After slowly evaporating the solvents for several days, some light yellow single crystals were obtained.

\section{Experimental details}

The structure was solved by direct methods and refined with the SHELX crystallographic software package [1]. The hydrogen atoms were placed at calculated positions and refined as riding atoms.

\section{Comment}

Cancer is one of the most health problems in the world; although many classes of drugs were used for the treatment, the needs for safe and effective anticancer compounds are still significant target. Heterocyclic building blocks represent useful scaffolds for applications in medicinal chemistry. To our knowledge, 4-anilinopyrimidine derivatives often have a wide range of biological activities, especially in antitumor drugs $[4,5]$. Some of them have been developed as the important prescription antitumor drugs in the market, for example, Pazopanib [6], Ceritinib [7], Brigatinib [8], afatinib [9] and rociletinib [10] are all based on 4-anilinopyrimidine.

The title molecule (see the Figure) has a benzofuro [2,3- $d]$ pyrimidine skeleton, in which all bond lengths and bond angles fall in normal ranges [11]. The dihedral angles between benzofuro[2,3- $d$ ]pyrimidine and two phenyl rings are $62.46^{\circ}$ and $20.25^{\circ}$ respectively. And the two phenyl planes are not coplanar with a dihedral angle of $64.08^{\circ}$. Furthermore, a mass of the intermolecular hydrogen bonds $\mathrm{N}(3)-\mathrm{H}(3 \mathrm{~A}) \cdots \mathrm{N}(4), \mathrm{N}(3)-\mathrm{H}(3 \mathrm{~A}) \cdots \mathrm{N}(4)$, $\mathrm{C}(14)-\mathrm{H}(14) \cdots \mathrm{N}(1), \mathrm{C}(4)-\mathrm{H}(4) \cdots \mathrm{O}(3)$ and $\mathrm{C}(11)-\mathrm{H}(11) \cdots$ $\mathrm{O}(4)$ are found in the title structure. It is worth noting that the crystal packing is further stabilized by weak p-p interactions $(3.5142 \AA)$. These interactions together with intermolecular hydrogen bond result in the formation of a three-dimensional framework.

Author contributions: All the authors have accepted responsibility for the entire content of this submitted manuscript and approved submission.

Research funding: None declared.

Conflict of interest statement: The authors declare no conflicts of interest regarding this article. 


\section{References}

1. Agilent Technologies. CrysAlis ${ }^{\mathrm{PRO}}$ Software system (version 1.171.35.15); Agilent Technologies UK Ltd: Oxford, UK, 2011.

2. Sheldrick G. M. SHELXTL - Integrated space-group and crystal-structure determination. Acta Crystallogr. 2015, A71, 3-8.

3. Sheldrick G. M. Crystal structure refinement with SHELXL. Acta Crystallogr. 2015, C71, 3-8.

4. Abd E. H. S. R., Lasheen D. S., Hassan M. A., Abouzid K. A. M. Design and synthesis of 4-anilinothieno[2,3-d]pyrimidine-based compounds as dual EGFR/HER-2 inhibitors. Arch. Pharm. 2016, 349, 827-847.

5. Ding H.-G., Cai Z.-Q., Hou L., Hu Z.-Q., Jin Z.-S., Xu D., Cao H., Meng M.-M., Xie Y.-H., Zheng D.-Q. Synthesis and evaluation of some novel 6-substituted quinazoline derivatives as antitumor agents. J. Chem. Soc. Pakistan 2014, 87, 782-793.

6. Ghith A., Youssef K. M., Ismail N. S. M., Abouzid K. A. M. Design, synthesis and molecular modeling study of certain VEGFR-2 inhibitors based on thienopyrimidne scaffold as cancer targeting agents. Bioorg. Chem. 2019, 83, 111-128.

7. Sailaja T. G. G., Laxminarayanaa E., Chary M. T., Ramesh M. Synthesis and antibacterial activity of novel $4-\{4-($ methylamino) thieno[3,2-d]pyrimidin-2-yl\}-benzohydrazide derivatives. Russ. J. Gen. Chem. 2017, 87, 1275-1280.

8. Mohan C., Bhargava G., Bedi P. M. S. Thieno[3,2-d] pyrimidin4-one derivatives as potential antibacterial agents. J. Life Sci. 2009, 2, 97-101.

9. Kang D.-W., Fang Z.-G., Li Z.-Y., Huang B.-S., Zhang H., Lu X.-Y., Xu H.-R., Zhou Z.-G., Ding X., Daelemans D., Clercq E. D., Pannecouque C., Zhan P., Liu X.-Y. Design, synthesis, and evaluation of thiophene[3,2- $d$ ] pyrimidine derivatives as HIV 1 non-nucleoside reverse transcriptase inhibitors with significantly improved drug resistance profiles. J. Med. Chem. 2016, 59, 7991-8007.

10. Kang D.-W., Fang Z.-G., Huang B.-S., Lu X.-Y., Zhang H., Xu H.-R., Huo Z.-P., Zhou Z.-G., Yu Z., Meng Q., Wu G.-C., Ding X., Tian Y., Daelemans D., Clercq E. D., Pannecouque C., Zhan P., Liu X.-Y. Structure-based optimization of thiophene[3,2-d]pyrimidine derivatives as potent HIV 1 non-nucleoside reverse transcriptase inhibitors with improved potency against resistance-associated variants. J. Med. Chem. 2017, 60, 4424-4443.

11. Sheng J., Liu Z., Yan M., Zhang X., Wang D., Xu J., Zhang E., Zou Y. Biomass-involved synthesis of $N$-substituted benzofuro[2,3- $d$ ] pyrimidine-4-amines and biological evaluation as novel EGFR tyrosine kinase inhibitors. Org. Biomol. Chem. 2017, 15, 4971-4977. 